\title{
COMMENT
}

Check for updates

\section{Age-old musings: twenty-first century management of advanced kidney disease in older individuals}

Sarbjit Vanita Jassal ${ }^{凶}$ and Elbert Chow

Quality geriatric kidney care extends beyond traditional therapy to care that balances the impact of both disease and treatment around how individuals manage their daily routines. In this Comment, we discuss clinical and policy changes that could benefit older people with advanced kidney disease.

Starting dialysis often sharply increases the number and type of symptoms or problems experienced

Division of Nephrology, University Health Network, Toronto, ON, Canada.

凶e-mail: Vanita.Jassal@ uhn.ca

https://doi.org/10.1038 s41581-021-00511-6
Innovations in the field of geriatric nephrology have focused on clinical programs that incorporate elements of the Comprehensive Geriatric Assessment into the routine nephrology workflow. Many of these programs depend on geriatricians and allied health professionals being integrated into the nephrology team, or on nephrology team members willing to undergo additional training. Most programs capture key elements at baseline such as changes in LifeSpace (a useful functional measure of changes in the geographical area in which an individual lives and conducts their activities) $)^{1}$, the number of non-disease-specific impairments, frailty assessments and a recent history of accidental falls. Geriatric nephrology clinics occur largely in the outpatient setting and act as small pockets of specialized kidney care for older people, with a focus on comprehensive assessment and kidney health planning. A few of these programs extend to specialist provision of rehabilitation care ${ }^{2,3}$, but few extend into inpatient care or to older patients experiencing severe acute kidney injury.

Patients starting dialysis when aged $>70$ years face an uncertain health trajectory. The initial few months are associated with clinical events that disrupt home routines, which in turn cause a chain of events that often change the role of the patient within their social environment. Adaptation to the treatments is difficult ${ }^{4}$. Paradoxically, starting dialysis often sharply increases the number and type of symptoms or problems experienced, particularly early in the dialysis trajectory. Patients often report the substitution of one symptom for another: for example, walking might become easier as volume overload is controlled with dialysis and the effort of breathing decreases, but simultaneous changes, such as post-dialysis fatigue or dizziness, may alter the desire and motivation to be active. Moreover, dialysis-related symptoms that are often regarded as minor, such as shoulder discomfort caused by maintaining the arm used for vascular access in a fixed position or knee stiffness caused by prolonged sitting, might alter how easily a patient completes a dressing or toileting task. In some individuals these changes lead to loss of independence, and patients can enter a spiral of decline soon after starting dialysis. Observational studies confirm that, in addition to high rates of early mortality and hospitalization after dialysis initiation, patients with a high baseline burden of problems not related to kidney disease, such as falls, and cognitive or mobility issues, have a high risk of functional dependency or need for long-term care ${ }^{5}$.

The COVID-19 pandemic has emphasized the need for a pragmatic approach to how we provide care. The blunt truth is that, for most older patients with kidney failure, life is short, even with optimal treatment planning. Barring the few who have a realistic chance of transplantation, most older patients entering the kidney failure trajectory face a steady decline in health, punctuated by acute hospitalizations, regardless of treatment choices. The public are more attuned to how non-disease-specific problems, such as cognitive decline or accidental falls, have a higher prognostic value than a simple count of comorbidities ${ }^{6}$. Patients increasingly understand that health extends beyond survival and with that come opportunities for a paradigm shift in geriatric kidney care. Although most older patients starting dialysis experience the rapid and severe decline described above, it is those few who thrive beyond expectation, respond well to rehabilitation and blossom with dialysis care that the clinician sees repeatedly and likely the reason we paint dialysis as an easier life than it often is. It may be that these few patients are seen so often that clinicians are most likely to remember them, creating doubt and uncertainty about beneficence and harm in our minds. Likely, it is our own uncertainty that influences our choice of wording, and consequently what the patient hears, sees and chooses.

Not all patients choose to start dialysis. Several centres have reported that $10-20 \%$ of patients opt for a conservative approach to their kidney care 7 . To deliver comprehensive conservative kidney care, clinicians 
Beneficence could be weighed in terms of tradeoffs between different life and symptom experiences work together to provide fastidious attention to symptoms and intensification of oral, subcutaneous and, occasionally, intravenous medications as needed to minimize these symptoms. This approach also involves timely de-prescribing of medications that are unlikely to bring benefit over the coming weeks or months of life, such as statins and renin-angiotensin-aldosterone system inhibitors. Through rationalization of medications, relaxed dietary restrictions and priority given to care that has immediate benefits, patients and families experience a health trajectory that differs from that often associated with dialysis care. Comprehensive kidney care includes support from community nursing and allied health, resulting in improved wellbeing and emotional health. Of note, the difference in longevity between patients receiving dialysis-based and conservative kidney care seems to be small, likely measured in weeks to months ${ }^{8}$.

Much has changed over the last decade, but the provision of high-quality geriatric kidney care still faces many challenges. We believe that certain jargon commonly used and accepted on nephrology units should be banished. Conservative kidney care, so often described as "no treatment" or "gone palliative", could be reframed as intensification of oral and subcutaneous medications. In many cases, dialysis should be likened to other palliative treatments, such as a palliative radiotherapy used to shrink an enlarging neck tumour, and described as a treatment used to reduce the initial threat of death, such as that posed by hyperkalaemia, only to have the threat recur within days. Beneficence could be weighed in terms of trade-offs between different life and symptom experiences, avoiding its interpretation as a choice between life or death and between discomfort or comfort. Providers might deliver a prognosis by focusing on goal setting for tasks that are achievable over weeks and extend to months, rather than those projected over years, and increasingly adopt terminology such as "entered ... final days", “...final weeks” or “...final months” of life.

Policy leaders also have an opportunity, and duty, to mitigate financial barriers to high-quality geriatric kidney care. For example, in many countries, patients and families are expected to carry the costs of medications, even those occurring at the end of life ${ }^{8}$. The nephrology community should aim to extend medication coverage for patients managed on a conservative kidney care pathway so that it is comparable to that of individuals starting dialysis. Other opportunities for financial change include a formal review of the impact on geriatric kidney care of linking dialysis unit reimbursement payments to quality-of-care targets. At present, frail, older patients are often expected to meet the same quality targets for clearance, phosphate and vascular access as patients in whom dialysis is a conduit to transplant surgery. Adjustment of quality standards to realistic outcome expectations might allow clinicians to adapt care, for example, by relaxing dietary restrictions for individuals who wish to continue on dialysis or avoiding arteriovenous graft surgeries in those in the final stage of life. Lastly, with data emerging that physician and care provider attitudes directly impact the patterns of treatment provided $^{9,10}$, it might be timely to address the delicate subject of physician reimbursement and to ensure that physician remuneration for conservative kidney care aligns more with that of providing dialysis care.

Robust research is needed to better understand certain aspects of medical care that best fall under the term 'professionalism'. Perhaps nephrologists need to revive the art of describing not just the desired or intended outcome with dialysis, but a realistic outcome. Should we challenge ourselves to revert from providing a 'buffet' of all available treatments from which families and patients are to choose, to a 'well-curated menu' that is limited to a few suggested interventions most likely to benefit the patient?

1. Bowling, C. B. et al. Community mobility among older adults with reduced kidney function: a study of life-space. Am. J. Kidney Dis. 63, 429-436 (2014).

2. Sheshadri, A. $\&$ Johansen, K. L. Prehabilitation for the frail patient approaching ESRD. Semin. Nephrol. 37, 159-172 (2017).

3. Farragher, J. et al. Importance of early inpatient geriatric rehabilitation on outcomes in individuals on dialysis. Arch. Phys. Med. Rehabil. 101, 227-233 (2020).

4. Abel, D. L. Functioning on dialysis: an oxymoron? Clin. J. Am. Soc. Nephrol. 14, 963-964 (2019).

5. Jassal, S. V. et al. Functional dependence and mortality in the International Dialysis Outcomes and Practice Patterns Study (DOPPS). Am. J. Kidney Dis. 67, 283-292 (2016).

6. Aliberti, M. J. R. \& Avelino-Silva, T. J. Beyond age-improvement of prognostication through physical and cognitive functioning for nursing home residents with COVID-19. JAMA Intern. Med. 181, 448-449 (2021).

7. Morton, R. L. et al. Factors influencing patient choice of dialysis versus conservative care to treat end-stage kidney disease. CMAJ 184, E277-E283 (2012).

8. O'Connor, N. R. \& Kumar, P. Conservative management of end-stage renal disease without dialysis: a systematic review. J. Palliat. Med. 15, 228-235 (2012)

9. Jassal, S. V. et al. International variation in dialysis discontinuation in patients with advanced kidney disease. CMAJ 192, E995-E1002 (2020).

10. Lunney, M. et al. Availability, accessibility, and quality of conservative kidney management worldwide. Clin. J. Am. Soc. Nephrol. 16, 79-87 (2020).

Competing interests

The authors declare no competing interests. 